\title{
Rogosinski's lemma for univalent functions, hyperbolic Archimedean spirals and the Loewner equation
}

\author{
Oliver Roth and Sebastian Schleißinger \\ November 5, 2018
}

\begin{abstract}
We describe the region $\mathcal{V}\left(z_{0}\right)$ of values of $f\left(z_{0}\right)$ for all normalized bounded univalent functions $f$ in the unit disk $\mathbb{D}$ at a fixed point $z_{0} \in \mathbb{D}$. The proof is based on the radial Loewner differential equation. We also prove an analogous result for the upper half-plane using the chordal Loewner equation.
\end{abstract}

\section{Results: the unit disk}

We denote by $\mathbb{D}:=\{z \in \mathbb{C}:|z|<1\}$ the open unit disk in the complex plane $\mathbb{C}$ and by $\mathcal{H}_{0}(\mathbb{D})$ the set of all holomorphic functions $f: \mathbb{D} \rightarrow \mathbb{D}$ normalized by $f(0)=0$ and $f^{\prime}(0) \geq 0$. The Schwarz lemma tells us that $\left|f\left(z_{0}\right)\right| \leq\left|z_{0}\right|$ for any $f \in \mathcal{H}_{0}(\mathbb{D})$ and any $z_{0} \in \mathbb{D}$. In 1934 Rogosinski [11] (also [3, p. 200]) proved a far reaching sharpening of the Schwarz lemma by giving an explicit description of the region of values of $f\left(z_{0}\right)$, $f \in \mathcal{H}_{0}(\mathbb{D})$, at a fixed point $z_{0} \in \mathbb{D}$. In this note we prove an analogue of Rogosinski's result for univalent functions in $\mathcal{H}_{0}(\mathbb{D})$ by providing an explicit description of the regions of values

$$
\mathcal{V}\left(z_{0}\right):=\left\{f\left(z_{0}\right): f \in \mathcal{H}_{0}(\mathbb{D}) \text { univalent }\right\}, \quad z_{0} \in \mathbb{D}
$$

It turns out that the set $\mathcal{V}\left(z_{0}\right)$ admits a fairly appealing description in terms of hyperbolic geometry. In order to state the main results, we therefore endow the unit disk with the standard hyperbolic metric

$$
\lambda_{\mathbb{D}}(z) d z=\frac{2|d z|}{1-|z|^{2}}
$$

of constant curvature -1 , see [1]. The induced hyperbolic distance $\mathrm{d}_{\mathbb{D}}(z, w)$ between $z, w \in \mathbb{D}$ is then given by

$$
\mathrm{d}_{\mathbb{D}}(z, w)=\log \frac{1+\left|\frac{z-w}{1-\bar{w} z}\right|}{1-\left|\frac{z-w}{1-\bar{w} z}\right|}
$$

Theorem 1.1

Let $z_{0} \in \mathbb{D}$. Then

$$
\mathcal{V}\left(z_{0}\right) \cup\{0\}=\left\{z=|z| e^{i \varphi} \in \mathbb{D}: \mathrm{d}_{\mathbb{D}}(0, z)-\mathrm{d}_{\mathbb{D}}\left(0, z_{0}\right) \leq-\left|\varphi-\arg z_{0}\right|, \varphi \in \mathbb{R}\right\} .
$$



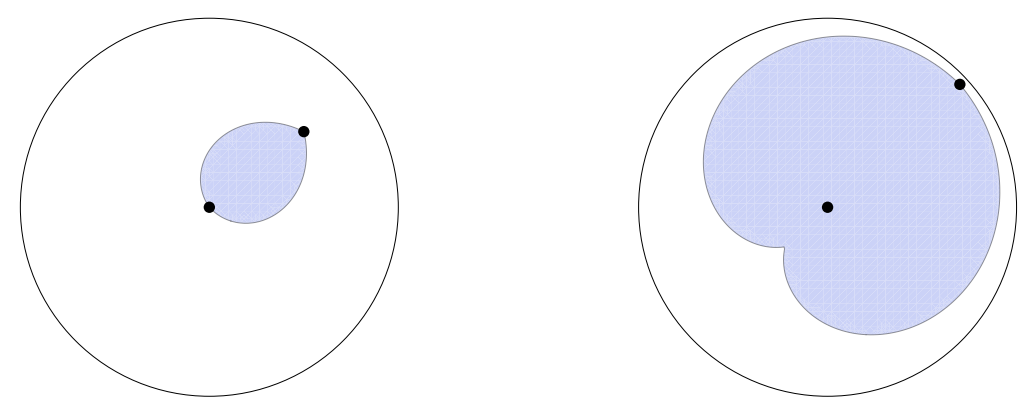

Figure 1: The set $\mathcal{V}\left(z_{0}\right)$ for $z_{0}=0.5+0.4 i$ (left) $z_{0}=0.7+0.65 i$ (right)
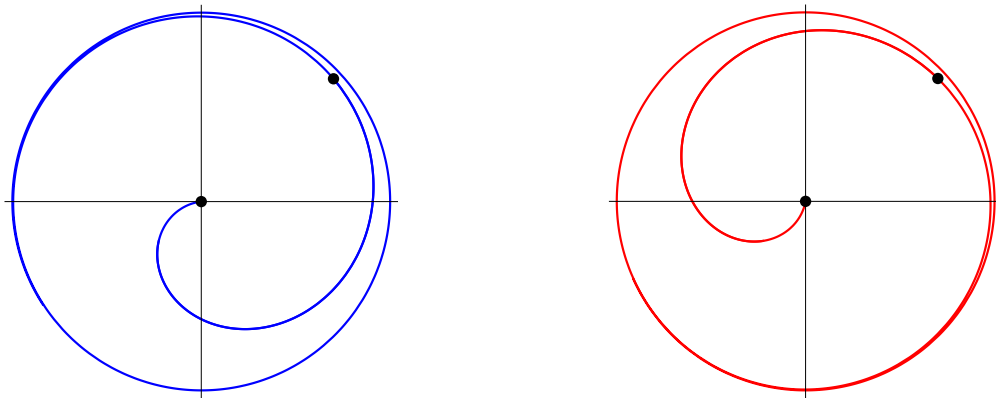

Figure 2: Hyperbolic Archimedean spirals $\mathrm{S}_{\mathrm{h}}^{+}\left(z_{0}\right)$ and $\mathrm{S}_{\mathrm{h}}^{-}\left(z_{0}\right)$ for $z_{0}=0.7+0.65 i$.

In particular, the boundary of the region of values $\mathcal{V}\left(z_{0}\right)$ is composed of parts of the two curves

$$
\begin{aligned}
& \mathrm{S}_{\mathrm{h}}^{+}\left(z_{0}\right):=\left\{|z| e^{i \varphi}: \mathrm{d}_{\mathbb{D}}(0, z)=+\varphi+\mathrm{d}_{\mathbb{D}}\left(0, z_{0}\right)-\arg z_{0}, \varphi \geq \arg z_{0}-\mathrm{d}_{\mathbb{D}}\left(0, z_{0}\right)\right\} \\
& \mathrm{S}_{\mathrm{h}}^{-}\left(z_{0}\right):=\left\{|z| e^{i \varphi}: \mathrm{d}_{\mathbb{D}}(0, z)=-\varphi+\mathrm{d}_{\mathbb{D}}\left(0, z_{0}\right)+\arg z_{0}, \varphi \leq \arg z_{0}+\mathrm{d}_{\mathbb{D}}\left(0, z_{0}\right)\right\}
\end{aligned}
$$

In complete analogy to the standard euclidean Archimedean spirals

$$
\begin{aligned}
& \mathrm{S}_{\mathrm{e}}^{+}\left(w_{0}\right)=\left\{|w| e^{i \varphi} \in \mathbb{C}:|w|=+\varphi+\left|w_{0}\right|-\arg w_{0}, \varphi \geq \arg w_{0}-\left|w_{0}\right|\right\} \\
& \mathrm{S}_{\mathrm{e}}^{-}\left(w_{0}\right)=\left\{|w| e^{i \varphi} \in \mathbb{C}:|w|=-\varphi+\left|w_{0}\right|+\arg w_{0}, \varphi \leq \arg w_{0}+\left|w_{0}\right|\right\}
\end{aligned}
$$

which pass through both the origin and the point $w_{0} \in \mathbb{C}$, we call the curves $\mathrm{S}_{\mathrm{h}}^{+}\left(z_{0}\right)$ and $\mathrm{S}_{\mathrm{h}}^{-}\left(z_{0}\right)$ the (standard) hyperbolic Archimedean spirals through the origin and the point $z_{0} \in \mathbb{D}$.

Now fix $z_{0} \in \mathrm{S}_{\mathrm{h}}^{ \pm}\left(z_{0}\right)$ and move towards the origin while staying on $\mathrm{S}_{\mathrm{h}}^{ \pm}\left(z_{0}\right)$. Stop either when you reach the point $z_{1}^{ \pm}$of hyperbolic distance $\mathrm{d}_{\mathbb{D}}\left(0, z_{0}\right)-\pi$ from the origin or when you reach the origin. In the first case, we see from the definition of $\mathrm{S}_{\mathrm{h}}^{ \pm}\left(z_{0}\right)$ that $\arg z_{1}^{ \pm}=\mp \pi+\arg z_{0}$, so $z_{1}:=z_{1}^{+}=z_{1}^{-}$. Let $z_{1}:=0$ in the second case. In both cases we define

$$
\gamma^{ \pm}\left(z_{0}\right):=\text { connected part of } \mathrm{S}_{\mathrm{h}}^{ \pm}\left(z_{0}\right) \text { between } z_{0} \text { and } z_{1} .
$$

\section{Corollary 1.2}

Let $z_{0} \in \mathbb{D}$. Then the region of values $\mathcal{V}\left(z_{0}\right)$ has the following properties.

(a) $\partial \mathcal{V}\left(z_{0}\right)=\gamma^{+}\left(z_{0}\right) \cup \gamma^{-}\left(z_{0}\right) \cup\{0\}$ and $\mathcal{V}\left(z_{0}\right) \cup\{0\}$ is a Jordan domain bounded by the Jordan curve $\gamma^{+}\left(z_{0}\right) \cup \gamma^{-}\left(z_{0}\right)$. 

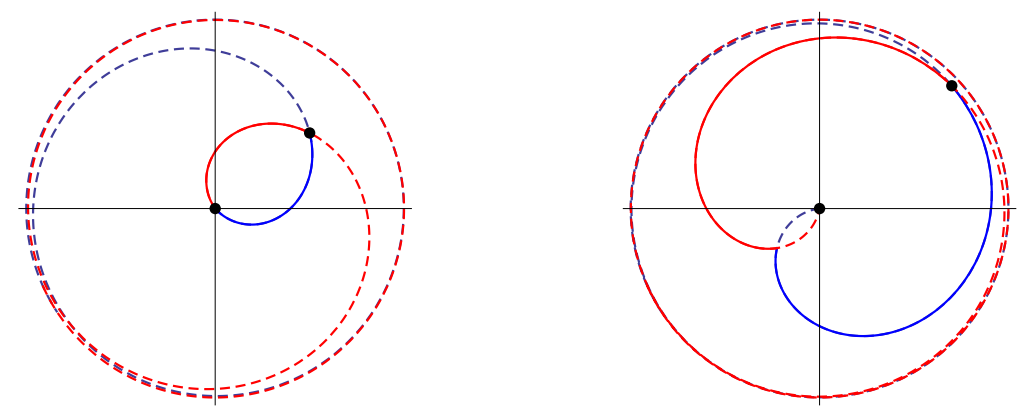

Figure 3: $\mathrm{S}_{\mathrm{h}}^{ \pm}\left(z_{0}\right)$ (dashed) and $\gamma^{ \pm}\left(z_{0}\right)$ (solid) in blue $(+)$ and in red $(-)$ for $z_{0}=$ $0.5+0.4 i$ (left) and for $z_{0}=0.7+0.65 i$ (right);

(b) The origin is an isolated boundary point of $\mathcal{V}\left(z_{0}\right)$ if and only if $\left|z_{0}\right|>$ $\tanh (\pi / 2)=0.917152 \ldots$

(c) $\mathcal{V}\left(z_{0}\right)$ is convex if and only if $\left|z_{0}\right| \leq \tanh (\pi / 4)=0.655794 \ldots 1^{1}$

(d) Each boundary curve $\gamma^{ \pm}\left(z_{0}\right)$ has hyperbolic length

$$
L_{h}\left(\gamma^{ \pm}\left(z_{0}\right)\right)= \begin{cases}\sinh \left(\mathrm{d}_{\mathbb{D}}\left(0, z_{0}\right)\right) & \text { if }\left|z_{0}\right| \leq \tanh (\pi / 2), \\ \sinh \left(\mathrm{d}_{\mathbb{D}}\left(0, z_{0}\right)\right)-\sinh \left(\mathrm{d}_{\mathbb{D}}\left(0, z_{0}\right)-\pi\right) & \text { if }\left|z_{0}\right| \geq \tanh (\pi / 2) .\end{cases}
$$

Theorem 1.1 as well as Corollary 1.2 will be proved in Section 3 by making use of the Loewner differential equation

$$
\begin{aligned}
\dot{w}(t) & =-w(t) \frac{\kappa(t)+w(t)}{\kappa(t)-w(t)}, \quad t \geq 0 \\
w(0) & =z_{0} \in \mathbb{D}
\end{aligned}
$$

with fixed $z_{0} \in \mathbb{D}$, and its reachable set

$$
\begin{aligned}
\mathcal{R}\left(z_{0}\right):=\{w(t): & w(\cdot) \text { is a solution to }(1.2) \text { for some } \\
& \text { continuous function } \kappa:[0, \infty) \rightarrow \partial \mathbb{D}, t \geq 0\}, \quad z_{0} \in \mathbb{D} .
\end{aligned}
$$

Note that by standard results of Loewner's theory it is clear that $\overline{\mathcal{V}\left(z_{0}\right)}=\overline{\mathcal{R}\left(z_{0}\right)}$. It will turn out that actually the stronger statement $\mathcal{V}\left(z_{0}\right)=\mathcal{R}\left(z_{0}\right)$ holds, so in order to prove Theorem 1.1 it suffices to describe the reachable set $\mathcal{R}\left(z_{0}\right)$. By definition, $\mathcal{R}\left(z_{0}\right)$ is made up of the trajectories of the Loewner equation (1.2). Any trajectory $w:[0, T] \rightarrow \mathbb{D}$ of the Loewner equation such that $w(T)$ is a boundary point of $\mathcal{R}\left(z_{0}\right)$ is therefore of special interest and is called an optimal trajectory. The corresponding "control function" $\kappa:[0, T] \rightarrow \partial \mathbb{D}$ is then called optimal.

\section{Remark 1.3 (The principle of optimality)}

The well-known principle of optimality asserts that if a control function $\kappa:[0, T] \rightarrow$ $\mathbb{D}$ ist optimal, then it is also optimal on $\left[0, T^{\prime}\right]$ for any $T^{\prime} \leq T$. This means that a trajectory $w:[0, T] \rightarrow \mathbb{D}$ of the Loewner equation which delivers at time $t=T$ a boundary point $w(T)$ of the reachable set $\mathcal{R}\left(z_{0}\right)$, then $w(t) \in \partial \mathcal{R}\left(z_{0}\right)$ for every $t \in[0, T]$. Hence every initial piece of an optimal trajectory is again optimal. This is a

\footnotetext{
${ }^{1}$ The number $\tanh (\pi / 4)$ is also the radius of starlikeness in the class $\mathcal{S}$ found by Grunsky 1934.
} 
general principle which holds for any control system under very general assumptions. It holds in particular for the Loewner equation and has been utilized in this context before e.g. by Friedland and Schiffer [4], Kirwan and Pell [9] and more recently by Graham, Hamada, Kohr and Kohr [5] for the Loewner equation in several complex variables.

The next result shows that for any $z_{0} \in \mathbb{D}$ the Loewner equation $(1.2)$ has exactly two optimal trajectories. These optimal trajectories parametrize the entire boundary of the reachable set $\mathcal{R}\left(z_{0}\right)$. They form exactly the $\operatorname{arcs} \gamma^{ \pm}\left(z_{0}\right)$ of the hyperbolic Archimedean spirals $\mathrm{S}_{\mathrm{h}}^{ \pm}\left(z_{0}\right)$.

\section{Theorem 1.4}

Let $z_{0} \in \mathbb{D}$. Then there exist two trajectories $w_{z_{0}}^{ \pm}:[0, \infty) \rightarrow \mathbb{D}$ such that $w_{z_{0}}^{ \pm}([0, \infty))$ is the connected part of $S_{\mathrm{h}}^{ \pm}\left(z_{0}\right)$ between $z_{0}$ and 0 .

(a) If $\mathrm{d}_{\mathbb{D}}\left(0, z_{0}\right) \leq \pi$, then $w_{z_{0}}^{ \pm}:[0, \infty) \rightarrow \mathbb{D}$ is optimal for every $t>0$ and $w_{z_{0}}^{ \pm}([0, \infty))=\gamma^{ \pm}\left(z_{0}\right) \backslash\{0\}$.

(b) If $\mathrm{d}_{\mathbb{D}}\left(0, z_{0}\right)>\pi$, then $w_{z_{0}}^{ \pm}:[0, T] \rightarrow \mathbb{D}$ is optimal for every $0<T \leq T_{\max }\left(z_{0}\right)$, but not optimal for any $T>T_{\max }\left(z_{0}\right)$. Here

$$
T_{\text {max }}\left(z_{0}\right)=-\log \left[\frac{\sinh \left(\mathrm{d}_{\mathbb{D}}\left(0, z_{0}\right)-\pi\right)}{\sinh \left(\mathrm{d}_{\mathbb{D}}\left(0, z_{0}\right)\right)}\right] .
$$

Furthermore, $w_{z_{0}}^{ \pm}\left(\left[0, T_{\max }\left(z_{0}\right)\right]\right)=\gamma^{ \pm}\left(z_{0}\right)$.

\section{Remark 1.5}

Theorem 1.1 has a very well-known classical counterpart for the class

$$
\mathcal{S}:=\left\{f: \mathbb{D} \rightarrow \mathbb{C} \text { univalent, } f(0)=0, f^{\prime}(0)=1\right\}
$$

established by Grunsky 1932, see [7], who proved the remarkable fact that the set

$$
\mathcal{W}\left(z_{0}\right):=\left\{\log \left(f\left(z_{0}\right) / z_{0}\right): f \in \mathcal{S}\right\}
$$

is exactly the disk

$$
\left\{w \in \mathbb{C}:\left|w-\log \frac{1}{1-\left|z_{0}\right|^{2}}\right| \leq \log \frac{1+\left|z_{0}\right|}{1-\left|z_{0}\right|}\right\} .
$$

Grunsky's result was extended e.g. by Gorjainov and Gutljanski [6], who obtained a precise description of the sets

$$
\mathcal{W}^{M}\left(z_{0}\right):=\left\{\log \frac{f\left(z_{0}\right)}{z_{0}}: f \in \mathcal{S} \text { such that }|f(z)|<M \text { for every } z \in \mathbb{D}\right\}
$$

for any $M>1$. The results in [7, 6] are much more difficult to prove than the results of the present paper since the sets $\mathcal{W}\left(z_{0}\right)$ and $\mathcal{W}^{M}\left(z_{0}\right)$ have in fact a much more complicated structure than the set $\mathcal{V}\left(z_{0}\right)$. In principle, the set $\mathcal{V}\left(z_{0}\right)$ can certainly be described using the results in [6] about the sets $\mathcal{W}^{M}\left(z_{0}\right)$. The purpose of the present paper is to give a simple and direct proof of the simple structure of the set $\mathcal{V}\left(z_{0}\right)$ emphasizing some of its remarkable hyperbolic geometric properties without making appeal to the deeper results about the sets $\mathcal{W}\left(z_{0}\right)$ and $\mathcal{W}^{M}\left(z_{0}\right)$ due to Grunsky [7] and Gorjainov and Gutljanski [6]. 


\section{Results: The upper half-plane}

We now replace the interior normalization $f(0)=0$ and $f^{\prime}(0) \geq 0$, which was used throughout section 11, by an appropriate boundary condition. For this purpose, it is convenient to use the upper half-plane $\mathbb{H}:=\{z \in \mathbb{C}: \operatorname{Im} z>0\}$ with its distinguished boundary point $\infty$ instead of the unit disk $\mathbb{D}$. We consider the set $\mathcal{H}_{\infty}(\mathbb{H})$ of all holomorphic functions $g: \mathbb{H} \rightarrow \mathbb{H}$ such that the so-called hydrodynamic normalization,

$$
g(z)-z \rightarrow 0 \text { as } z \rightarrow \infty \text { in } S_{\beta}:=\{z \in \mathbb{C}:|\arg z-\pi / 2|<\beta\}, 0<\beta<\pi / 2,
$$

is satisfied.

\section{Remark 2.1}

The hydrodynamic normalization for functions in $\mathcal{H}_{\infty}(\mathbb{H})$ corresponds to the interior normalization for functions in $\mathcal{H}_{0}(\mathbb{D})$ in the following way. First note that the interior condition $f(0)=0$ and $f^{\prime}(0) \geq 0$ for every $f \in \mathcal{H}_{0}(\mathbb{D})$ enforces that the only conformal automorphism of $\mathbb{D}$ contained in $\mathcal{H}_{0}(\mathbb{D})$ is the identity. Now, every function $g \in \mathcal{H}_{\infty}(\mathbb{H})$ has an angular derivative at $z=\infty$,

$$
g^{\prime}(\infty):=\angle \lim _{z \rightarrow \infty} \frac{g(z)}{z}=1
$$

and hence the angular limit

$$
g(\infty):=\angle \lim _{z \rightarrow \infty} g(z)=\infty
$$

Note that the two boundary conditions $g(\infty)=\infty$ and $g^{\prime}(\infty)=1$ alone allow for infinitely many conformal automorphisms $z+b, b \in \mathbb{R}$, of $\mathbb{H}$, but the sharper hydrodynamic condition for $\mathcal{H}_{\infty}(\mathbb{H})$ guarantees that the only conformal automorphism of $\mathbb{H}$ contained in $\mathcal{H}_{0}(\mathbb{H})$ is the identity.

The following result is the analogue of Theorem 1.1 for the upper half-plane.

\section{Theorem 2.2}

Let $z_{0} \in \mathbb{H}$. Then

$$
\left\{g\left(z_{0}\right): g \in \mathcal{H}_{\infty}(\mathbb{H}) \text { univalent }\right\}=\left\{z \in \mathbb{C}: \operatorname{Im} z>\operatorname{Im} z_{0}\right\} \cup\left\{z_{0}\right\} .
$$

\section{Remark 2.3}

Using the well-known Nevanlinna representation for holomorphic functions in $\mathbb{H}$ with positive imaginary part (see [12, Theorem 5.3]), it is immediate that

$$
\left\{g\left(z_{0}\right): g \in \mathcal{H}_{\infty}(\mathbb{H})\right\} \subseteq\left\{z \in \mathbb{C}: \operatorname{Im} z>\operatorname{Im} z_{0}\right\} \cup\left\{z_{0}\right\}
$$

Hence, Theorem 2.2 tells us that the set of values $g\left(z_{0}\right)$ for all univalent functions $g \in \mathcal{H}_{\infty}(\mathbb{H})$ is the same as the set of values $g\left(z_{0}\right)$ for all $g \in \mathcal{H}_{\infty}(\mathbb{H})$. This is a significant difference to the unit disk case, where a comparison of Theorem 1.1 with Rogosinski's Lemma shows that the set $\mathcal{V}\left(z_{0}\right)$ of values $f\left(z_{0}\right)$ for all univalent functions $f \in \mathcal{H}_{0}(\mathbb{D})$ is strictly smaller than the set of values $f\left(z_{0}\right)$ for all functions $f \in \mathcal{H}_{0}(\mathbb{D})$. 
As in the unit disk case, the proof of Theorem 2.2 relies on the Loewner differential equation, but now we have to use the chordal version

$$
\begin{aligned}
\dot{w}(t) & =\frac{-2}{w(t)-U(t)}, \quad t \geq 0, \\
w(0) & =z_{0} \in \mathbb{H},
\end{aligned}
$$

with fixed $z_{0} \in \mathbb{H}$, and its reachable set

$$
\begin{aligned}
\mathcal{R}\left(z_{0}\right):=\{w(t): & w(\cdot) \text { is a solution to } 2.1) \text { for some } \\
& \text { continuous function } U:[0, \infty) \rightarrow \mathbb{R}, t \geq 0\}, \quad z_{0} \in \mathbb{H} .
\end{aligned}
$$

It is well-known (see [10, Chapter 4] and [2, 8]) that every solution $g_{t}$ of the chordal Loewner equation

$$
\dot{g}_{t}(z)=\frac{-2}{g_{t}(z)-U(t)}, \quad g_{0}(z)=z
$$

generated by a continuous driving function $U:[0, \infty) \rightarrow \mathbb{R}$ is a univalent function on $\mathbb{H}$ such that

$$
g_{t}(z)=z-\frac{b(t)}{z}+\mathcal{O}\left(1 / z^{2}\right) \quad \text { as } z \rightarrow \infty .
$$

In particular, $g_{t} \in \mathcal{H}_{\infty}(\mathbb{H})$ for every $t \geq 0$. Hence Remark 2.3 implies that Theorem 2.2 is an immediate consequence of the following result, which describes the reachable set $\mathcal{R}\left(z_{0}\right)$ for any $z_{0} \in \mathbb{H}$.

\section{Theorem 2.4}

Let $z_{0} \in \mathbb{H}$. Then $\mathcal{R}\left(z_{0}\right)=\left\{z \in \mathbb{H}: \operatorname{Im} z>\operatorname{Im} z_{0}\right\} \cup\left\{z_{0}\right\}$.

\section{Proofs: the unit disk}

The proofs are based on an extremely simple differential inequality for the "hyperbolic polar coordinates" of the solutions of Loewner's equation, which follows immediately from the particular form of the Loewner equation.

\subsection{The basic differential inequality}

Fix $z_{0} \in \mathbb{D}$ and let $w:[0, \infty) \rightarrow \mathbb{D}$ be the solution to the Loewner equation $(1.2)$ generated by a measurable function $\kappa:[0, \infty) \rightarrow \partial \mathbb{D}$, that is,

$$
\frac{d}{d t}\{\log w(t)\}=-\frac{\kappa(t)+w(t)}{\kappa(t)-w(t)}, \quad w(0)=z_{0} .
$$

Separation into real and imaginary parts and writing $w(t)=|w(t)| e^{i \varphi(t)}$ gives the equivalent pair of equations

$$
\frac{d}{d t}\{|w(t)|\}=-|w(t)| \frac{1-|w(t)|^{2}}{|\kappa(t)-w(t)|^{2}}
$$

and

$$
\frac{d}{d t}\{\varphi(t)\}=-2 \frac{\operatorname{Im}\{\overline{\kappa(t)} w(t)\}}{|\kappa(t)-w(t)|^{2}} .
$$


Using (1.1) we can rewrite 3.1 in the form

$$
\frac{d}{d t}\left\{\mathrm{~d}_{\mathbb{D}}(0, w(t))\right\}=\frac{d}{d t}\left\{\log \frac{1+|w(t)|}{1-|w(t)|}\right\}=-2 \frac{|w(t)|}{|\kappa(t)-w(t)|^{2}} .
$$

Now using the simple inequality

$$
|w(t)| \geq \pm \operatorname{Im}\{\overline{\kappa(t)} w(t)\}
$$

we combine 3.2 and 3.3 and arrive at the differential inequality

$$
\frac{d}{d t}\left\{\mathrm{~d}_{\mathbb{D}}(0, w(t)\} \leq \pm \frac{d}{d t}\{\varphi(t)\}\right.
$$

3.2 Integrating the basic differential inequalities and proof of Theorem 1.1 Integrating (3.5) yields

$$
\mathrm{d}_{\mathbb{D}}(0, w(T))-\mathrm{d}_{\mathbb{D}}\left(0, z_{0}\right) \leq-\left|\varphi(T)-\arg z_{0}\right|, \quad T \in[0, \infty),
$$

with equality if

$$
\frac{d}{d t}\left\{\mathrm{~d}_{\mathbb{D}}(0, w(t))\right\}=+\frac{d}{d t}\{\varphi(t)\} \leq 0 \quad \text { for all } t \in[0, T]
$$

or if

$$
\frac{d}{d t}\left\{\mathrm{~d}_{\mathbb{D}}(0, w(t))\right\}=-\frac{d}{d t}\{\varphi(t)\} \leq 0 \quad \text { for all } t \in[0, T]
$$

This shows that

$$
\mathcal{R}\left(z_{0}\right) \subseteq\left\{w=|w| e^{i \varphi} \in \mathbb{D}: \mathrm{d}_{\mathbb{D}}(0, w)-\mathrm{d}_{\mathbb{D}}\left(0, z_{0}\right) \leq-\left|\varphi-\arg z_{0}\right|, \varphi \in \mathbb{R}\right\} .
$$

We now show that this inclusion is sharp. We first consider Case I. Then equality holds in (3.6) in this case, i.e.,

$$
\mathrm{d}_{\mathbb{D}}(0, w(T))-\mathrm{d}_{\mathbb{D}}\left(0, z_{0}\right)=\varphi(T)-\arg z_{0},
$$

and equality holds in (3.4) with + for every $t \in[0, T]$. This means that

$$
\overline{\kappa(t)} w(t)=i|w(t)| \quad \text { for every } t \in[0, T] .
$$

Therefore, 3.3 reduces to

$$
\frac{d}{d t}\left[\log \frac{1+|w(t)|}{1-|w(t)|}\right]=-2 \frac{|w(t)|}{1+|w(t)|^{2}}
$$

or

$$
\frac{d}{d t}\left\{\mathrm{~d}_{\mathbb{D}}(0, w(t))\right\}=-\tanh \left(\mathrm{d}_{\mathbb{D}}(0, w(t))\right)
$$

and an integration leads to

$$
\mathrm{d}_{\mathbb{D}}(0, w(t))=\operatorname{Arcsinh}\left(e^{-t} \sinh \left(\mathrm{d}_{\mathbb{D}}\left(0, z_{0}\right)\right)\right) .
$$


In a similar way, (3.2) reduces to

and another integration gives

$$
\frac{d}{d t}\{\varphi(t)\}=-\tanh \left(\mathrm{d}_{\mathbb{D}}(0, w(t))\right)
$$

$$
\varphi(t)=\varphi^{+}(t):=\operatorname{Arcsinh}\left(e^{-t} \sinh \left(\mathrm{d}_{\mathbb{D}}\left(0, z_{0}\right)\right)\right)-\mathrm{d}_{\mathbb{D}}\left(0, z_{0}\right)+\arg z_{0} .
$$

Using (3.8), we see that $\kappa(t)=-i e^{i \varphi^{+}(t)}$ for every $t \in[0, T]$. We can now define

$$
\kappa^{+}(t):=-i e^{i \varphi^{+}(t)}, \quad t \in[0, \infty) .
$$

This gives a (real-analytic) control function $\kappa^{+}:[0, \infty) \rightarrow \partial \mathbb{D}$. The corresponding solution $w_{z_{0}}^{+}:[0, \infty) \rightarrow \mathbb{D}$ of the Loewner equation $(1.2)$ satisfies

$$
\mathrm{d}_{\mathbb{D}}\left(0, w_{z_{0}}^{+}(t)\right)-\mathrm{d}_{\mathbb{D}}\left(0, z_{0}\right)=\arg w_{z_{0}}^{+}(t)-\arg z_{0} \text { for all } t \geq 0
$$

by construction. Case II can be handled in a similar way. In fact, if we set

$$
\kappa^{-}(t):=-i e^{i \varphi^{-}(t)}
$$

where

$$
\varphi^{-}(t):=-\operatorname{Arcsinh}\left(e^{-t} \sinh \left(\mathrm{d}_{\mathbb{D}}\left(0, z_{0}\right)\right)\right)+\mathrm{d}_{\mathbb{D}}\left(0, z_{0}\right)+\arg z_{0}
$$

then the corresponding solution $w_{z_{0}}^{-}:[0, \infty) \rightarrow \mathbb{D}$ of the Loewner equation 1.2 satisfies

$$
\mathrm{d}_{\mathbb{D}}\left(0, w_{z_{0}}^{-}(t)\right)-\mathrm{d}_{\mathbb{D}}\left(0, z_{0}\right)=-\arg w_{z_{0}}^{-}(t)+\arg z_{0} \text { for all } t \geq 0 .
$$

This shows that the inclusion (3.7) is sharp. In order to conclude

$$
\mathcal{R}\left(z_{0}\right)=\left\{w=|w| e^{i \varphi} \in \mathbb{D}: \mathrm{d}_{\mathbb{D}}(0, w)-\mathrm{d}_{\mathbb{D}}\left(0, z_{0}\right) \leq-\left|\varphi-\arg z_{0}\right|, \varphi \in \mathbb{R}\right\} \backslash\{0\}
$$

we just note that $\mathcal{V}\left(z_{0}\right) \cup\{0\}$ is a compact subset of the complex plane, which is starlike with respect to the boundary point 0 (i.e., if $w \in \mathcal{V}\left(z_{0}\right)$, then $r w \in \mathcal{V}\left(z_{0}\right)$ for any $0 \leq r \leq 1)$ and that $\overline{\mathcal{R}\left(z_{0}\right)}=\overline{\mathcal{V}\left(z_{0}\right)}$ by Loewner's theory. This proves 3.11 and $\mathcal{R}\left(z_{0}\right)=\mathcal{V}\left(z_{0}\right)$. The proof of Theorem 1.1 is complete.

\subsection{Proof of Theorem 1.4}

We note that in view of $(3.9)$ and 3.10$)$ the two trajectories $w_{z_{0}}^{ \pm}([0, \infty))$ form the connected parts of the hyperbolic Archimedean spirals $\mathrm{S}_{\mathrm{h}}^{ \pm}\left(z_{0}\right)$ between $z_{0}$ and 0 . These two trajectories never meet if and only if $\varphi^{+}(t)-\arg z_{0}>-\pi$ and $\varphi^{-}(t)-$ $\arg z_{0}<\pi$ for every $t>0$, which happens if and only if $\mathrm{d}_{\mathbb{D}}\left(0, z_{0}\right) \leq \pi$. In this case, we thus have $w_{z_{0}}^{ \pm}([0, \infty))=\gamma^{ \pm} \backslash\{0\}$. If $\mathrm{d}_{\mathbb{D}}\left(0, z_{0}\right)>\pi$, then the two trajectories $w_{z_{0}}^{ \pm}$ first meet at time $t=T_{\max }\left(z_{0}\right)$, where $\varphi^{+}\left(T_{\max }\left(z_{0}\right)\right)=-\pi+\arg z_{0}$, that is,

$$
\operatorname{Arcsinh}\left(e^{-T_{\max }\left(z_{0}\right)} \sinh \left(\mathrm{d}_{\mathbb{D}}\left(0, z_{0}\right)\right)\right)=\mathrm{d}_{\mathbb{D}}\left(0, z_{0}\right)-\pi
$$

i.e.,

$$
T_{\text {max }}\left(z_{0}\right)=-\log \left[\frac{\sinh \left(\mathrm{d}_{\mathbb{D}}\left(0, z_{0}\right)-\pi\right)}{\sinh \left(\mathrm{d}_{\mathbb{D}}\left(0, z_{0}\right)\right)}\right] .
$$

Clearly, $w_{z_{0}}^{ \pm}$is not optimal on $[0, T]$ for any $T>T_{\max }\left(z_{0}\right)$ and $w_{z_{0}}^{ \pm}\left(\left[0, T_{\max }\left(z_{0}\right)\right]\right)=$ $\gamma^{ \pm}\left(z_{0}\right)$. This proves Theorem 1.4 . 


\subsection{Proof of Corollary 1.2}

(a) is clear from the above discussion. (b) The origin is an isolated boundary point of $\mathcal{V}\left(z_{0}\right)$ if and only if $\mathrm{d}_{\mathbb{D}}\left(0, z_{0}\right)>\pi$, that is, $\left|z_{0}\right|>\tanh (\pi / 2)$. (c) $\mathcal{V}\left(z_{0}\right)$ is convex if and only if $\varphi^{+}(t)-\arg z_{0} \geq-\pi / 2$ and $\varphi^{-}(t)-\arg z_{0} \leq \pi / 2$ for every $t>0$. This occurs if and only if $\mathrm{d}_{\mathbb{D}}\left(0, z_{0}\right) \leq \pi / 2$, that is, $\left|z_{0}\right| \leq \tanh (\pi / 4)$.

(d) In hyperbolic polar coordinates the curve $\gamma^{+}\left(z_{0}\right)$ has the parametrization $I \ni$ $\varphi \mapsto|\gamma(\varphi)| e^{i \varphi}$, where $\mathrm{d}_{\mathbb{D}}(0, \gamma(\varphi))=\varphi+\mathrm{d}_{\mathbb{D}}\left(0, z_{0}\right)-\arg z_{0}$ and

$$
I:= \begin{cases}{\left[\arg z_{0}-\mathrm{d}_{\mathbb{D}}\left(0, z_{0}\right), \arg z_{0}\right]} & \text { if } \mathrm{d}_{\mathbb{D}}\left(0, z_{0}\right) \leq \pi, \\ {\left[\arg z_{0}-\pi, \arg z_{0}\right]} & \text { if } \mathrm{d}_{\mathbb{D}}\left(0, z_{0}\right)>\pi .\end{cases}
$$

Now, a computation shows

$$
2 \frac{\left|\gamma^{\prime}(\varphi)\right|}{1-|\gamma(\varphi)|^{2}}=\frac{1+|\gamma(\varphi)|^{2}}{1-|\gamma(\varphi)|^{2}}=\cosh \left(\mathrm{d}_{\mathbb{D}}(0, \gamma(\varphi))\right)
$$

so

$$
\begin{aligned}
L_{h}\left(\gamma^{+}\left(z_{0}\right)\right) & =\int_{I} 2 \frac{\left|\gamma^{\prime}(\varphi)\right|}{1-|\gamma(\varphi)|^{2}} d \varphi=\int_{I} \cosh \left(\varphi+\mathrm{d}_{\mathbb{D}}\left(0, z_{0}\right)-\arg z_{0}\right) d \varphi \\
& = \begin{cases}\sinh \left(\mathrm{d}_{\mathbb{D}}\left(0, z_{0}\right)\right) & \text { if } \mathrm{d}_{\mathbb{D}}\left(0, z_{0}\right) \leq \pi \\
\sinh \left(\mathrm{d}_{\mathbb{D}}\left(0, z_{0}\right)\right)-\sinh \left(\mathrm{d}_{\mathbb{D}}\left(0, z_{0}\right)-\pi\right) & \text { if } \mathrm{d}_{\mathbb{D}}\left(0, z_{0}\right)>\pi\end{cases}
\end{aligned}
$$

\section{Proofs: the upper half-plane}

Fix $z_{0} \in \mathbb{H}$ and let $H\left(z_{0}\right):=\left\{z \in \mathbb{H}: \operatorname{Im} z>\operatorname{Im} z_{0}\right\} \cup\left\{z_{0}\right\}$. In order to prove Theorem 2.4, we first note that for every $g \in \mathcal{H}_{\infty}(\mathbb{H})$

$$
\operatorname{Im} g\left(z_{0}\right) \geq \operatorname{Im} z_{0} \quad \text { with equality if and only if } g(z)=z
$$

This follows immediately from the Poisson representation

$$
\operatorname{Im} g(z)=c \operatorname{Im} z+\frac{\operatorname{Im} z}{\pi} \int_{-\infty}^{\infty} \frac{d \mu(t)}{(t-\operatorname{Re} z)^{2}+(\operatorname{Im} z)^{2}},
$$

where $\mu$ is a nonnegative Borel measure on $\mathbb{R}$ such that

$$
\int_{-\infty}^{\infty} \frac{d \mu(t)}{1+t^{2}}<\infty
$$

$($ see $[12])$ and

$$
c=\lim _{y \rightarrow \infty} \operatorname{Im} g(i y) / y,
$$

i.e., $c=1$ for $f \in \mathcal{H}_{\infty}(\mathbb{H})$. Clearly, (4.1) shows that $\mathcal{R}\left(z_{0}\right) \subseteq H\left(z_{0}\right)$.

Now let $z \in H\left(z_{0}\right)$. We need to find a driving function $U$ such that the solution $w(t)$ of (2.1) passes through $z$. We separate into real and imaginary parts and write 
$w(t)=x(t)+i y(t)$ and $z_{0}=x_{0}+i y_{0}$. Now, we claim that $U$ can be chosen such that $w(t)$ connects $z_{0}$ and $z$ by a straight line segment, i.e.

$$
x(t)=c \cdot y(t)+x_{0}-c \cdot y_{0}
$$

where

$$
c=\frac{\operatorname{Re} z-\operatorname{Re} z_{0}}{\operatorname{Im} z-\operatorname{Im} z_{0}}
$$

In order to prove this, we separate equation (2.1) into real and imaginary parts and obtain

$$
\dot{x}(t)=\frac{2(U(t)-x(t))}{(U(t)-x(t))^{2}+y(t)^{2}}, \quad \dot{y}(t)=\frac{2 y(t)}{(U(t)-x(t))^{2}+y(t)^{2}},
$$

with initial conditions $x(0)=x_{0}$ and $y(0)=y_{0}$. We now assume that $x(t)$ and $y(t)$ are related by

$$
U(t)-x(t)=c \cdot y(t) .
$$

Then we get the following initial value problem:

$$
\dot{x}(t)=\frac{2 c}{\left(1+c^{2}\right) y(t)}, \quad \dot{y}(t)=\frac{2}{\left(1+c^{2}\right) y(t)}, \quad x(0)=x_{0}, y(0)=y_{0},
$$

which can be solved directly:

$$
y(t)=\sqrt{\frac{4}{1+c^{2}} t+y_{0}^{2}} \quad \text { and } \quad x(t)=c y(t)+x_{0}-c y_{0}, \quad t \geq 0 .
$$

Hence if we now define

$$
U(t):=c y(t)+x(t)=2 c \sqrt{\frac{4}{1+c^{2}} t+y_{0}^{2}}+x_{0}-c y_{0}
$$

then by construction the solution $w(t)=x(t)+i y(t)$ of $(2.1)$ satisfies $x(t)=c y(t)+$ $x_{0}-c y_{0}$ In particular, the trajectory $t \mapsto w(t)$ is the halfline starting at $z_{0}$ through the point $z$, so $z \in \mathcal{R}\left(z_{0}\right)$. This completes the proof of Theorem 2.4 .

\section{References}

[1] A. Beardon and D. Minda, The Hyperbolic Metric and Geometric Function Theory, in: S. Ponnusamy, T. Sugawa and M. Vuorinen (editors), Quasiconformal Mappings and their Applications, Narosa 2007.

[2] M. Contreras, S. Díaz-Madrigal and P. Gumenyuk, Geometry behind chordal Loewner chains, Complex Anal. Oper. Theory 4 No. 3 (2010), 541-587.

[3] P. L. Duren, Univalent functions, Springer 1983.

[4] S. Friedland and M. Schiffer, On coefficient regions of univalent functions, J. Analyse Math. 31 (1977), 125-168.

[5] I. Graham, H. Hamada, G. Kohr and M. Kohr, Extreme points, support points and the Loewner variation in several complex variabels, Sci. China Math. 55 (2012), no. 7, 1353-1366. 
[6] G. G. Gorjajnov and V. Ja. Gutljanski, On extremal problems in the class $S_{M}, M a t . S b$., Kiev (1976), 242-246.

[7] H. Grunsky, Neue Abschätzungen zur konformen Abbildung ein- und mehrfach zusammenhängender Bereiche, Schr. Math. Inst. u. Inst. Angew. Math. Univ. Berlin 1 (1932), 95-140.

[8] P. Gumenyuk and A. del Monaco, Chordal Loewner equation, arXiv:1302.0898v2

[9] W. E. Kirwan and G. Schober, New inequalities from old ones, Math. Z. 180 (1982), 19-40.

[10] G. Lawler, Conformally invariant Processes in the Plane, Amer. Math. Soc. 2005.

[11] W. Rogosinski, Zum Schwarzschen Lemma, Jber. Deutsche Math.-Verein. 44 (1934), 258-261.

[12] M. Rosenblum and J. Rovnyak, Topics in Hardy Classes and Univalent Functions, Birkhäuser 1994.

Oliver Roth

Sebastian Schleißinger

Department of Mathematics

University of Würzburg

97074 Würzburg

Germany

roth@mathematik.uni-wuerzburg.de

sebastian.schleissinger@mathematik.uni-wuerzburg.de 